\title{
Electronic and magnetic properties of TTF and TCNQ covered Co thin films
}

Elmer van Geijn, Kai Wang, and Michel P. de Jong

Citation: J. Chem. Phys. 144, 174708 (2016); doi: 10.1063/1.4948413

View online: http://dx.doi.org/10.1063/1.4948413

View Table of Contents: http://aip.scitation.org/toc/jcp/144/17

Published by the American Institute of Physics 


\title{
Electronic and magnetic properties of TTF and TCNQ covered Co thin films
}

\author{
Elmer van Geijn, a) Kai Wang, and Michel P. de Jong \\ NanoElectronics Group, MESA+ Institute for Nanotechnology, University of Twente, P.O. Box 217, \\ 7500 AE Enschede, The Netherlands
}

(Received 22 December 2015; accepted 19 April 2016; published online 5 May 2016)

\begin{abstract}
Interfacial effects like orbital hybridization and charge transfer strongly influence the transfer of spins from ferromagnetic metals to organic semiconductors and can lead to the formation of interfacial states with distinct magnetic properties. The changes in the electronic and magnetic properties of a thin Co film upon adsorption of a layer of either the molecular organic electron donor tetrathiafulvalene (TTF) or the acceptor tetracyanoquinodimethane (TCNQ) have been investigated by X-ray absorption spectroscopy and X-ray magnetic circular dichroism using synchrotron radiation. Clear differences between the spectra of the adsorbed molecules and the neutral molecules show the hybridization of the molecular orbitals with the Co interface. Deposition of both organic materials leads to a small increase of the ratio of the orbital magnetic moment to the spin magnetic moment of the $\mathrm{Co}$ atoms at the interface. The main effect of overlayer deposition is a modification of the magnetic hysteresis of the Co film: The TCNQ slightly reduces the coercivity of the Co, while the TTF increases the coercivity by a factor of $\sim 1.5$. These complementary effects of either a molecular organic electron donor or acceptor on the interfacial properties of a metal ferromagnetic thin film are a promising result for the controlled modification of the magnetic structure of hybrid interfaces. Published by AIP Publishing. [http://dx.doi.org/10.1063/1.4948413]
\end{abstract}

\section{INTRODUCTION}

The field of organic spintronics offers the prospect of combining the advantageous properties of organic materials with those of spintronic phenomena. Spin-based applications using inorganic materials have shown remarkable physical phenomena such as giant magnetoresistance, ${ }^{1,2}$ tunnelling magnetoresistance, ${ }^{3}$ spin Hall effect, ${ }^{4-6}$ and spin-transfer torque. ${ }^{7}$ Several of these phenomena have found useful applications in everyday consumer electronics. It seems a logical step to combine the possibilities that spintronics has to offer with the flexibility, affordability, ease of manufacturing, and other advantages that the use of organic materials has brought to the field of electronics. The transport of charges and spins in organic semiconductors by hopping between the $\pi$-conjugated orbitals localized on different molecules (or polymer chain segments) is very different from the mechanism that applies for inorganic semiconductors, where charge carriers reside in fully delocalized bands. Theoretical models that agree with experimental results ${ }^{8}$ and provide insight into the underlying principles are still being developed. ${ }^{9,10}$ While spin polarized transport in organic semiconductors has been studied for more than a decade ${ }^{11}$ conclusive proof that spin-conserving transport is possible in organic materials has been elusive. ${ }^{8}$ Recently, spin-pumping experiments have been reported that show spin transport in organics to be feasible. ${ }^{12}$

Not only the transport mechanism of spins or spinpolarized charges is an important topic within organic

\footnotetext{
a) Author to whom correspondence should be addressed. Electronic mail: e.vangeijn@utwente.nl
}

spintronics but also the injection of spins from a ferromagnetic metal into an organic semiconductor is far from trivial. At the interface between the materials, phenomena like orbital hybridization and charge transfer can greatly influence the transport of spins across the interface. Spin-polarized microscopy and spectroscopy techniques have shown these effects to be typically spin dependent, resulting in a hybrid interface with magnetic properties that are quite different from those of the bulk ferromagnet. ${ }^{13-19}$ It is a topic of active study how this effects the spin-transport across the interface. ${ }^{20-24}$ In addition, the magnetic properties, such as the coercive field of the saturation magnetization, of ferromagnetic thin films may be tuned by the same interfacial effects. ${ }^{25,26}$ Very interestingly, it even turns out to be possible to induce ferromagnetism in thin diamagnetic $\mathrm{Cu}$ layers by interface formation with $\mathrm{C}_{60}$ molecules. ${ }^{27} \mathrm{~A}$ growing body of density functional theory based studies is starting to provide insight into the physics at play at these interfaces and hint at the exciting possibility of actively tailoring the properties of hybrid systems. ${ }^{28-30}$

In this study, we investigate the changes in the magnetic properties of Co thin films when forming an interface with organic molecules. Since charge redistribution is expected to be a possible cause of these changes, we chose both a strong electron acceptor, tetracyanoquinodimethane (TCNQ), and a strong electron donor, tetrathiafulvalene (TTF), as the organic adsorbents. By investigating the effects of these complementary molecular species, we shed light on the question whether systematic control of the interfacial magnetic properties through charge redistribution is feasible.

By recording X-ray absorption spectroscopy (XAS) spectra before and after deposition of the organic molecules, we can study the changes in the electronic properties of 
a ferromagnetic Co thin film. The impact on the magnetic properties of these changes can be probed by X-ray magnetic circular dichroism (XMCD) using circularly polarized X-rays.

\section{MATERIALS AND METHODS}

All sample preparation steps and experiments were performed in situ at the I1011 beam line at the MAX-lab synchrotron radiation facility in Lund, Sweden.

Single crystal sapphire (0001) was used as a substrate because Co will readily grow as an epitaxial fcc (111) film when deposited onto the clean sapphire surface at room temperature. ${ }^{31,32}$ The Co was evaporated onto the substrates under ultra-high vacuum (UHV) conditions from an e-beam evaporator. The base pressure of the system varied from $9.0 \times 10^{-9}$ to $1.5 \times 10^{-8}$ mbar during the experiments. We judged the thickness of the Co layers by the opacity of the film and estimated it to be between 10 and $20 \mathrm{~nm}$ for all samples.

The organic molecules (TCNQ and TTF) were deposited using a custom-made sublimation cell. The organic material (in powder form) was loaded into a small halogen light bulb, which we had cut open at the top, and sublimated by resistively heating the tungsten filament fitted inside the bulb. The sublimation cell was separated from the deposition chamber by a gate valve in order to prevent unwanted deposition of the organic material. For TTF, especially, this was essential as we found it to sublime fast enough for deposition at room temperature without any applied heating. To increase the sticking probability for TTF to the Co surface, the sample was cooled to liquid nitrogen temperature during TTF deposition. Deposition of TCNQ was carried out with the substrate held at room temperature.

In the used setup, the samples could be heated by a resistive heater mounted on the sample stage or cooled by a liquid nitrogen filled cold finger connected to the sample stage. A thermocouple connected to the sample stage gave an approximate reading of the sample temperature.

The X-rays for XAS and XMCD measurements were generated using an undulator that is inserted into the synchrotron storage ring. The elliptically polarizing undulator allowed for the use of X-rays with a degree of circularity of about $90 \%$. The sample was mounted at an angle of $60^{\circ}$ between the sample normal and the incoming photon beam. To correct for these experimental parameters, the magnetic moments extracted from the XMCD spectra were multiplied by $1 /\left(0.9 \times \sin 60^{\circ}\right) \approx 1.28$. During measurements, the sample sat between a pair of water-cooled copper coils that could generate magnetic fields up to 650 Oe. The sample could be transferred back and forth between deposition and measurement chamber without breaking UHV conditions.

$\mathrm{X}$-ray absorption spectra were recorded in the total electron yield (TEY) mode by measuring the drain current from the sample to ground while varying the incident photon energy. To ensure good electrical contact between the deposited Co films and the sample holder, we deposited a strip of tungsten along one edge of our sapphire substrates by magnetron sputtering prior to the experiments. By taking the difference of a pair of XAS spectra recorded with opposite relative orientations of the sample magnetization and light polarization, an XMCD spectrum was obtained, providing insight into the orbital and spin magnetic moments of the sample.

To be able to quantitatively compare the different recorded XAS spectra, these were corrected for changes in the intensity of the incident photon beam. To measure this intensity, a gold grid was placed in the path of the photon beam before it hit the sample. The drain current from this grid is proportional to the intensity of the photon beam. By taking the ratio of the sample drain current over the gold-grid drain current, $I_{0}$, the spectra are normalized for changes in the incoming beam.

Magnetization loops could be recorded by tuning the photon energy to the maximum of the $L_{3}$ peak of the Co $L$-edge and varying the external magnetic field. This method provides magnetization values relative to the absorption peak height, so no actual magnetization values.

\section{RESULTS AND DISCUSSION}

\section{TCNQ on Co}

For the first set of measurements, a film of TCNQ is deposited on a fcc Co film. The sample is at room temperature during all depositions and measurements. The deposition of the TCNQ layer is confirmed by the appearance of a strong $\mathrm{N} 1 s$ ( $K$-edge) absorption peak (Figure 1(a)). The features in the $\mathrm{N} K$-edge XAS spectrum of TCNQ adsorbed on Co are distinctly different from those of the neutral molecule, ${ }^{33}$ which is indicative of chemisorption and thus hybridization of electronic states. The $\mathrm{N}(1 s) \rightarrow \pi^{*}$ peak at the onset of the $K$-edge near $395 \mathrm{eV}$ clearly exhibits two split components. The corresponding feature of the neutral molecule is a single asymmetric peak and has been attributed to excitations into the

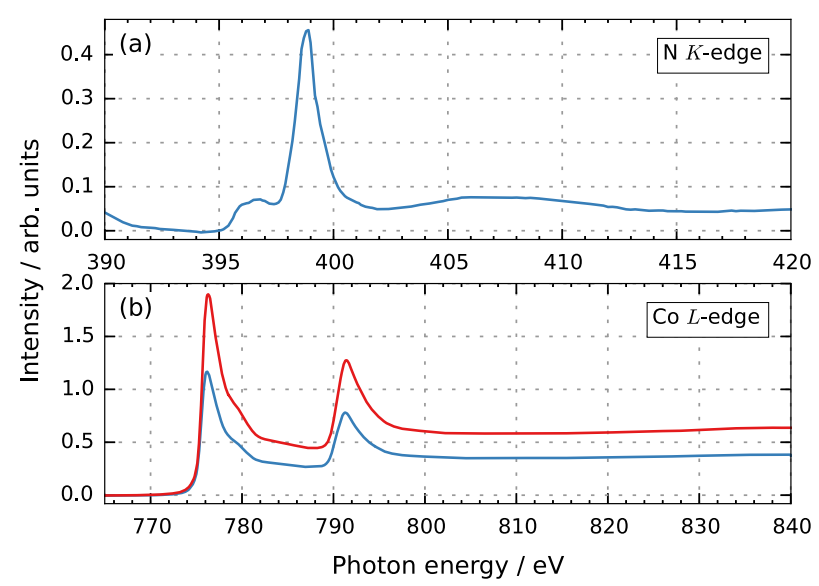

FIG. 1. N $K$-edge (a) and Co $L$-edge (b) for the clean Co thin film (red curve) and after deposition of a layer of TCNQ on top of the Co (blue curves). The spectra are normalized using reference spectra recorded with the gold grid and the pre-edge intensity is subtracted. Both the presence of the N $K$-edge peak and the attenuation of the Co $L$-edge peak demonstrate the formation of a TCNQ film. Comparison of the recorded $\mathrm{N} K$-edge with that of the neutral TCNQ molecule ${ }^{33}$ shows clear differences indicative of chemisorption. 


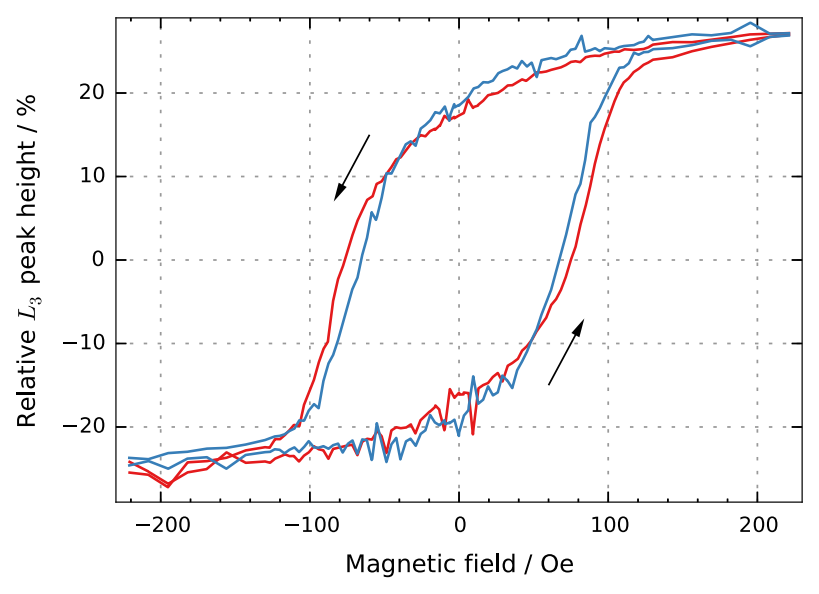

FIG. 2. Magnetic field sweep of the intensity of the Co $L_{3}$ peak for the clean Co thin film (red curve) and after deposition of a layer of TCNQ on top of the Co (blue curve). The latter exhibits a slightly lower coercivity and higher squareness. The arrows indicate the sweep direction of the magnetic field.

nearly degenerate $a_{u}$ and $b_{1 u} \pi^{*}$ orbitals (where the symmetry labels refer to $D_{2 h}$ symmetry). ${ }^{33}$ For the neutral molecule, a small peak associated with the $b_{2 g} \pi^{*}$ orbital is present on the high excitation energy side of the main $\mathrm{N}(1 s) \pi \rightarrow *$ resonance, which is absent for TCNQ on Co. Since TCNQ is a strong electron acceptor, it is expected that electrons have been transferred from Co to TCNQ at the interface.

The presence of the TCNQ layer also leads to the attenuation of the Co $2 p$ ( $L$-edge) absorption spectrum (Figure 1(b)). The amount of attenuation can be used to give an estimate of the thickness of the deposited inorganic layer. Based on measurements of the attenuation of TEY XAS peaks by overlayers, ${ }^{34,35}$ we estimate the thickness of the organic film to be around $0.5 \mathrm{~nm}$.

Due to the spin orbit coupling of the Co $2 p$ electronic states, the transition probabilities at the $L_{2,3}$-edge depend on the relative orientations of the magnetization of the sample and the helicity vector of the incoming light. By sweeping the magnetization while keeping the photon polarization fixed and at an energy matching the maximum of the $L_{3}$-edge of Co, the magnetic hysteresis curve of Figure 2 is measured. The deposition of TCNQ slightly lowers the coercivity of the $\mathrm{Co}$ and increases the squareness (remanence at zero applied field relative to saturation magnetization).

The XMCD spectra of the Co L-edge (Figure 3) show negligible change after TCNQ deposition. Sum rule analysis of the spectra ${ }^{36}$ gives a ratio of the orbital contribution to the spin contribution for the angular momentum of 0.051 for the clean Co thin film and 0.056 for the film with TCNQ. Due to the structure in the background after the absorption edge, it is difficult to determine quantitative values for the respective spin and orbital angular momenta. Our data suggest a value between 2.0 and $2.3 \mu_{\mathrm{B}}$ per atom for the spin angular momentum and between 0.10 and $0.13 \mu_{\mathrm{B}} /$ atom for the orbital angular momentum. An overview of all calculated magnetic moments is given in Table I. Note that the different values for $m_{\text {orb }} / m_{\text {spin }}$ of the two clean Co films in Table I might be related to a difference in film thickness in the range $10-20 \mathrm{~nm}$, such that a different surface-to-bulk ratio of the magnetic moments is observed in the TEY-mode XAS measurements.
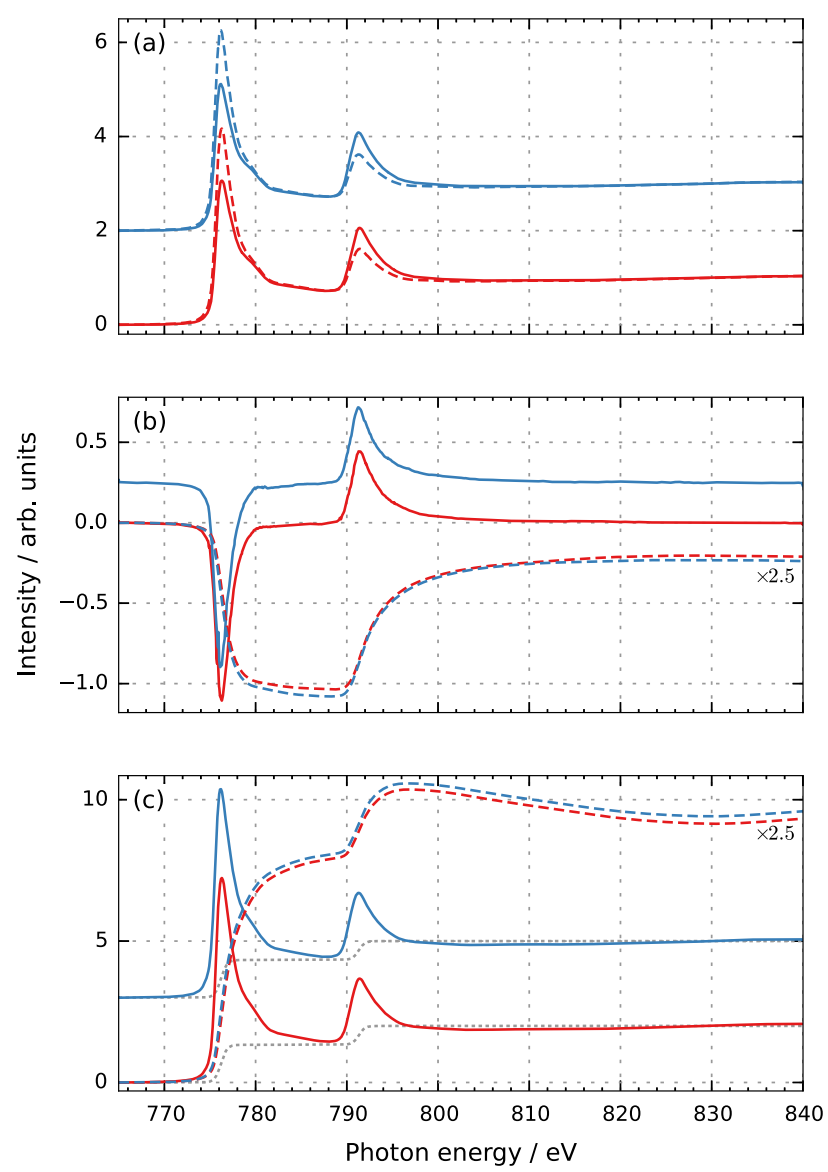

FIG. 3. Co $L$-edge XAS and XMCD spectra before (red curves) and after (blue curves; vertical offset for clarity) deposition of TCNQ. (a) Measured XAS spectra for opposite relative orientations of sample magnetization and photon polarization (solid and dashed lines). To do proper XMCD analysis, the pre-edge intensity is subtracted from the spectra, and the intensity at high photon energy far beyond the edge, where magnetic effects are absent, is normalized to unity. (b) XMCD spectra, difference between XAS spectra in (a) (solid), and integrated XMCD spectra (dashed; scaled down by a factor of 2.5 for clarity). (c) Summed XAS spectra (solid) and integrated sum spectra (dashed; scaled down by a factor of 2.5 for clarity) after subtraction of two-step-like edge-jump background (dotted, grey line). The variation in the integrated intensity at high photon energy is an artefact of the background subtraction procedure, which is hampered by the structure in the background.

\section{TTF on Co}

After replacing the TCNQ sublimation cell with one filled with TTF, the readings of the mass spectrometer for residual gas analysis, mounted on the preparation chamber, confirmed that the TTF sublimation rate was sufficiently high for TTF deposition while keeping the source material at room temperature. The absence of any $\mathrm{C} K$-edge signal in the XAS spectrum (Figure 4) after exposing the Co surface, held at room temperature, to TTF vapour for a few minutes indicates a low sticking probability of the molecules to the surface. To achieve TTF deposition, the sample is cooled, using liquid nitrogen, to approximately $100 \mathrm{~K}$ during exposure to the TTF vapour. The appearance of a clear $\mathrm{C} K$-edge signal affirms that a layer of TTF is indeed deposited on the surface under these conditions.

The C $K$-edge absorption shows a double main peak, whereas the neutral molecule shows a single peak. ${ }^{33}$ This 
TABLE I. Overview of magnetic moments calculated by sum rule analysis ${ }^{36}$ of the measured XMCD spectra. All values are in units of $\mu_{\mathrm{B}} /$ atom. Our data show an increase of the ratio of the orbital magnetic moment to the spin magnetic moment after deposition of both TCNQ and TTF on a clean Co thin film.

\begin{tabular}{lccc}
\hline \hline & $m_{\text {orb }} / m_{\text {spin }}$ & $m_{\text {orb }}$ & $m_{\text {spin }}$ \\
\hline TCNQ on Co & & & \\
\hline Clean Co film & 0.051 & $0.10-0.12$ & $2.0-2.3$ \\
TCNQ on Co & 0.056 & $0.11-0.13$ & $2.0-2.3$ \\
TTF on Co & & & \\
\hline Clean Co film (293 K) & 0.081 & $0.14-0.15$ & $1.7-1.8$ \\
Clean Co film (100 K) & 0.065 & $0.11-0.12$ & $1.6-1.8$ \\
TTF on Co (100 K) & 0.099 & $0.15-0.16$ & $1.5-1.6$ \\
\hline \hline
\end{tabular}

splitting is attributed to hybridization of the Co surface with the different molecular orbitals that contribute to this peak.

For the most prominent contribution of interface effects to the measured spectra, a TTF film thickness of a single monolayer would be ideal. Charge transfer between the Co and the TTF molecules at the interface is expected to lead to significant binding of these molecules. By gradually heating the sample, we aimed to remove all TTF except for the more strongly bound first monolayer. After heating the sample to $283 \mathrm{~K}$ for several minutes, the reduced film thickness leads to attenuation of the $\mathrm{C}$ peak. After subsequent heating to $323 \mathrm{~K}$, the TTF seems to be completely removed from the sample.

The magnetic hysteresis of the Co is strongly influenced by the deposition of a layer of TTF (Figure 5). The coercivity is increased by a factor of 1.5 and the magnetization saturates more gradually. After heating to $323 \mathrm{~K}$, the magnetic

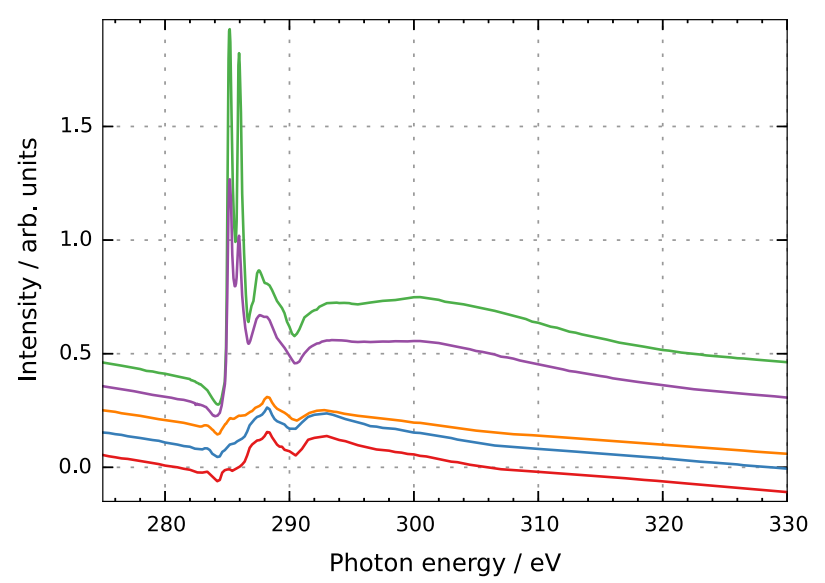

FIG. 4. XAS spectrum of the C $K$-edge of TTF covered Co (vertical offset for clarity). The red and blue curves for the clean Co surface, respectively, before and after exposure to TTF vapour at room temperature are very similar, indicating no noteworthy deposition of TTF. The structure at the C $K$-edge in these spectra results from adventitious carbon on the beam line optics, which is only partially corrected for by the $I_{0}$ measurement. Clear peaks are observed after deposition with the sample cooled down to about $100 \mathrm{~K}$ (green curve). After heating to $283 \mathrm{~K}$, the peaks are attenuated (magenta curve), indicating partial desorption of TTF. After heating of the sample to $323 \mathrm{~K}$ for about 5 min (orange curve), the spectrum resembles that of the clean Co thin film, suggesting that the TTF layer is completely removed.

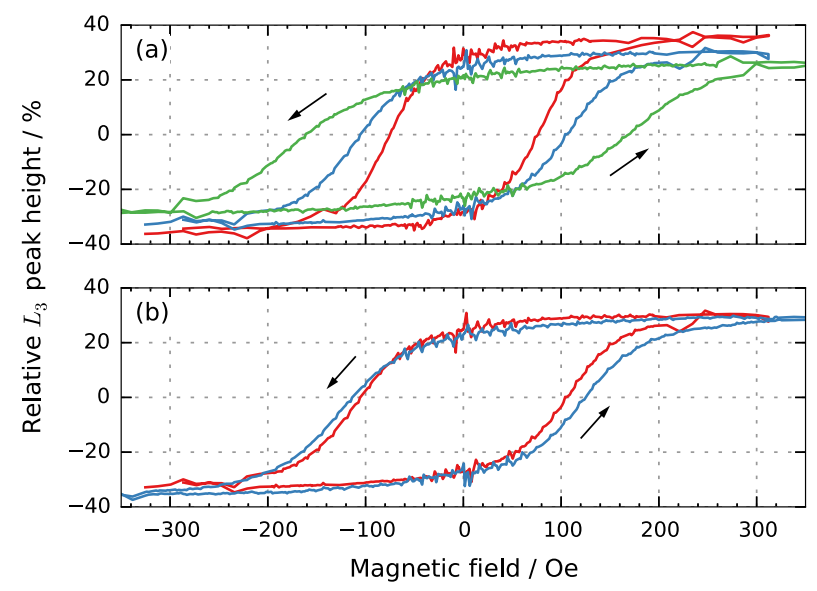

FIG. 5. Magnetic hysteresis of the Co $L_{3}$ XAS peak height. (a) Hysteresis for the clean Co thin film measured at room temperature (red curve), at $100 \mathrm{~K}$ (blue curve), and after deposition of a layer of TTF at $100 \mathrm{~K}$ (green curve). The TTF layer clearly increases the coercivity and reduces the squareness of the Co film. (b) Hysteresis curve measured at $100 \mathrm{~K}$ before TTF deposition (red curve) and after TTF deposition and subsequent heating to $323 \mathrm{~K}$ for 5 min (blue curve). After removal of the TTF layer, the magnetic hysteresis of the Co film reverts to its original shape.

behaviour reverts to that of the clean Co film, showing once more that the TTF layer is removed.

The XMCD analysis of the absorption spectra (Figure 6) shows strong variations in the ratio of the orbital to the spin magnetic moment. For the clean Co film at room temperature, the ratio is 0.081 ; at $100 \mathrm{~K}, 0.065$; and after TTF deposition at $100 \mathrm{~K}, 0.099$. For the actual magnetic moments, the data give a value between 0.14 and $0.15 \mu_{\mathrm{B}} /$ atom for the orbital contribution of the clean film at room temperature and between 1.7 and $1.8 \mu_{\mathrm{B}} /$ atom for the spin contribution. Measured at $100 \mathrm{~K}$, both moments decrease to between 0.11 and $0.12 \mu_{\mathrm{B}} /$ atom for the orbital part and between 1.6 and $1.8 \mu_{\mathrm{B}} /$ atom for the spin. After deposition of TTF, the orbital moment increases again to between 0.15 and $0.16 \mu_{\mathrm{B}} /$ atom and the spin moment decreases to between 1.5 and $1.6 \mu_{\mathrm{B}} /$ atom (see Table I for an overview). It should again be noted that these values are expected to have substantial errors due to the non-uniform background at the high energy side of the absorption edge. These errors are systematic for all measurements; therefore, the values can be used for qualitative comparisons between the different spectra.

The effects of TTF deposition on the magnetization reversal of the Co films are strong, while the changes in the XMCD spectra are subtle. The latter can in part be attributed to the finite probing depth of the TEY technique, meaning that only a small fraction of the Co $L$-edge signal stems from the outermost $\mathrm{Co}$ atoms that are primarily affected by bonding to TTF molecules. In addition, the effects of adsorption of molecules on ferromagnetic surfaces are strongly inhomogeneous, ${ }^{15,20}$ and an area averaged XMCD measurement is not representative of any significant, local modifications of surface magnetic moments. On the other hand, the magnetic anisotropy energy, which has a significant effect on the magnetic hysteresis loops, may be strongly affected by such local modifications. ${ }^{26}$ 

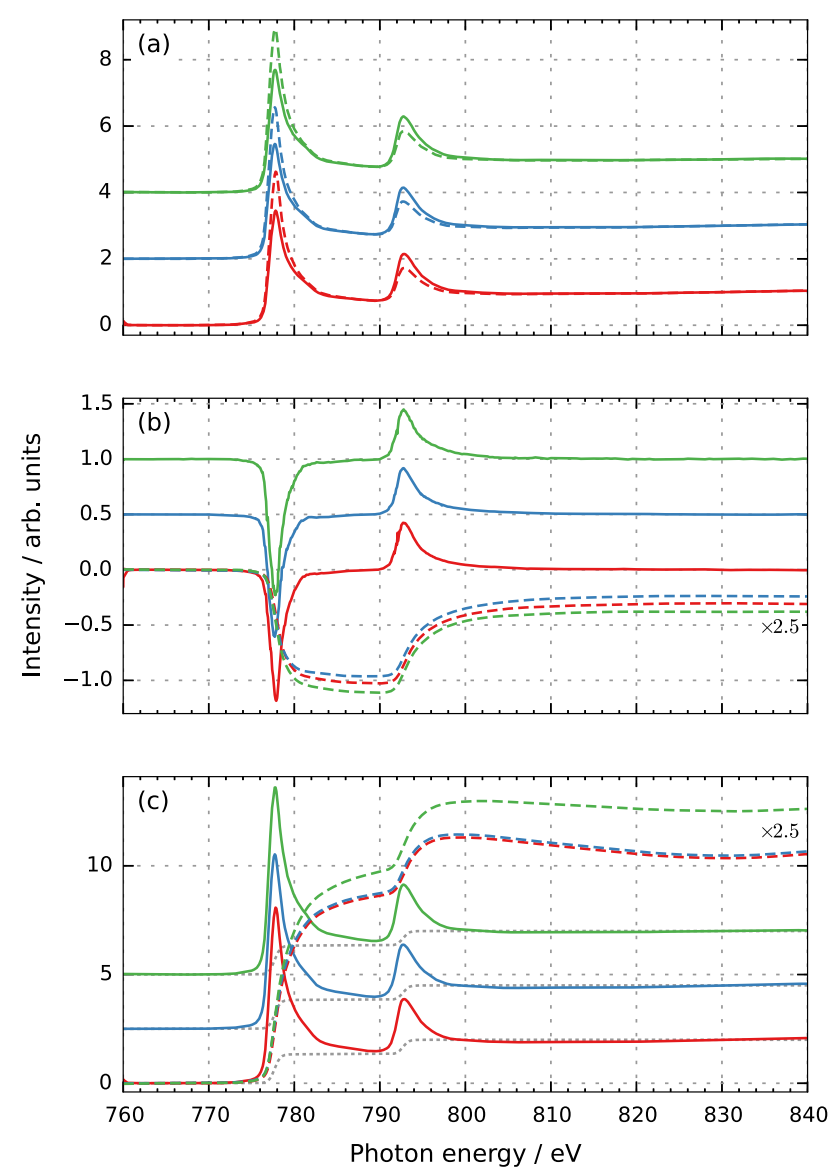

FIG. 6. Co $L$-edge XAS and XMCD spectra of the clean Co film measured at room temperature (red curves) and at $100 \mathrm{~K}$ (blue curves; vertical offset for clarity) and after deposition of TTF measured at $100 \mathrm{~K}$ (green curves; vertical offset for clarity). (a) Measured XAS spectra for opposite relative orientations of sample magnetization and photon polarization (solid and dashed lines). To do proper XMCD analysis, the pre-edge intensity is subtracted from the spectra, and the intensity at high photon energy far beyond the edge, where magnetic effects are absent, is normalized to unity. (b) XMCD spectra, difference between XAS spectra in (a) (solid), and integrated XMCD spectra (dashed; scaled down by a factor of 2.5 for clarity). (c) Summed XAS spectra (solid) and integrated sum spectra (dashed; scaled down by a factor of 2.5 for clarity) after subtraction of two-step-like edge-jump background (dotted, grey line). The variation in the integrated intensity at the high photon energy is an artefact of the background subtraction procedure, which is hampered by the structure in the background.

\section{CONCLUSION}

In summary, the XAS spectra for both TCNQ and TTF molecular layers on top of a ferromagnetic Co thin film have been measured. Hybridization of molecular orbitals with the Co interface can be inferred from clear differences between the recorded spectra and those of the respective neutral molecules.

$\mathrm{XMCD}$ analysis of the samples, using different relative orientations of sample magnetization and light polarization, suggests that both TCNQ and TTF lead to an increase of the ratio of the orbital magnetic moment to the spin magnetic moment.

Magnetic field sweeps of the Co $L_{3}$ peak intensity show that the coercivity of the Co film decreases slightly when the molecular electron acceptor TCNQ is deposited on the surface and increases with a factor of $\sim 1.5$ when the donor TTF is deposited on top. This complementary behaviour shows the possibility of controlled tuning of the properties of the Co interface by deposition of the suitable organic semiconductor.

\section{ACKNOWLEDGMENTS}

We acknowledge financial support from the European Research Council (ERC Starting Grant No. 280020) and the research program of the Foundation for Fundamental Research on Matter (FOM, Grant No. 10PR2808), which is part of the Netherlands Organization for Scientific Research (NWO).

${ }^{1}$ M. N. Baibich, J. M. Broto, A. Fert, F. N. Vandau, F. Petroff, P. Eitenne, G. Creuzet, A. Friederich, and J. Chazelas, Phys. Rev. Lett. 61, 2472 (1988).

${ }^{2}$ G. Binasch, P. Grünberg, F. Saurenbach, and W. Zinn, Phys. Rev. B 39, 4828 (1989).

${ }^{3}$ M. Julliere, Phys. Lett. A 54, 225 (1975).

${ }^{4}$ M. I. Dyakonov and V. I. Perel, JETP Letters-USSR 13, 467 (1971).

${ }^{5}$ J. E. Hirsch, Phys. Rev. Lett. 83, 1834 (1999).

${ }^{6}$ Y. K. Kato, R. C. Myers, A. C. Gossard, and D. D. Awschalom, Science 306, 1910 (2004).

${ }^{7}$ J. C. Slonczewski, J. Magn. Magn. Mater. 159, L1 (1996).

${ }^{8}$ A. Riminucci, M. Prezioso, C. Pernechele, P. Graziosi, I. Bergenti, R. Cecchini, M. Calbucci, M. Solzi, and V. A. Dediu, Appl. Phys. Lett. 102, 092407 (2013).

${ }^{9}$ N. J. Harmon and M. E. Flatte, Phys. Rev. B 90, 115203 (2014).

${ }^{10}$ Z. G. Yu, Phys. Rev. Lett. 111, 016601 (2013).

${ }^{11}$ Z. H. Xiong, D. Wu, Z. V. Vardeny, and J. Shi, Nature 427, 821 (2004).

${ }^{12}$ S. Watanabe, K. Ando, K. Kang, S. Mooser, Y. Vaynzof, H. Kurebayashi, E. Saitoh, and H. Sirringhaus, Nat. Phys. 10, 308 (2014).

${ }^{13}$ T. Suzuki, M. Kurahashi, X. Ju, and Y. Yamauchi, J. Phys. Chem. B 106, 11553 (2002).

${ }^{14}$ T. Suzuki, M. Kurahashi, X. Ju, and Y. Yamauchi, Surf. Sci. 549, 97 (2004).

${ }^{15}$ T. L. A. Tran, D. Cakir, P. K. J. Wong, A. B. Preobrajenski, G. Brocks, W. G. van der Wiel, and M. P. de Jong, ACS Appl. Mater. Interfaces 5, 837 (2013).

${ }^{16}$ A. L. Rizzini, C. Krull, A. Mugarza, T. Balashov, C. Nistor, R. Piquerel, S. Klyatskaya, M. Ruben, P. M. Sheverdyaeva, P. Moras, C. Carbone, C. Stamm, P. S. Miedema, P. K. Thakur, V. Sessi, M. Soares, F. Yakhou-Harris, J. C. Cezar, S. Stepanow, and P. Gambardella, Surf. Sci. 630, 361 (2014).

${ }^{17}$ F. Djeghloul, F. Ibrahim, M. Cantoni, M. Bowen, L. Joly, S. Boukari, P. Ohresser, F. Bertran, P. Le Fevre, P. Thakur, F. Scheurer, T. Miyamachi, R. Mattana, P. Seneor, A. Jaafar, C. Rinaldi, S. Javaid, J. Arabski, J. P. Kappler, W. Wulfhekel, N. B. Brookes, R. Bertacco, A. Taleb-Ibrahimi, M. Alouani, E. Beaurepaire, and W. Weber, Sci. Rep. 3, 1272 (2013).

${ }^{18}$ S. Lach, A. Altenhof, K. Tarafder, F. Schmitt, M. E. Ali, M. Vogel, J. Sauther, P. M. Oppeneer, and C. Ziegler, Adv. Funct. Mater. 22, 989 (2012).

${ }^{19}$ Z. Sun, Y. Zhan, S. Shi, and M. Fahlman, Org. Electron. 15, 1951 (2014).

${ }^{20}$ J. Brede, N. Atodiresei, S. Kuck, P. Lazic, V. Caciuc, Y. Morikawa, G. Hoffmann, S. Bluegel, and R. Wiesendanger, Phys. Rev. Lett. 105, 047204 (2010).

${ }^{21}$ K. V. Raman, A. M. Kamerbeek, A. Mukherjee, N. Atodiresei, T. K. Sen, P. Lazic, V. Caciuc, R. Michel, D. Stalke, S. K. Mandal, S. Bluegel, M. Muenzenberg, and J. S. Moodera, Nature 493, 509 (2013).

${ }^{22}$ S. L. Kawahara, J. Lagoute, V. Repain, C. Chacon, Y. Girard, S. Rousset, A. Smogunov, and C. Barreteau, Nano Lett. 12, 4558 (2012).

${ }^{23}$ S. Schmaus, A. Bagrets, Y. Nahas, T. K. Yamada, A. Bork, M. Bowen, E. Beaurepaire, F. Evers, and W. Wulfhekel, Nat. Nanotechnol. 6, 185 (2011).

${ }^{24}$ S. Steil, N. Grossmann, M. Laux, A. Ruffing, D. Steil, M. Wiesenmayer, S. Mathias, O. L. A. Monti, M. Cinchetti, and M. Aeschlimann, Nat. Phys. 9, 242 (2013).

${ }^{25}$ T. Moorsom, M. Wheeler, T. M. Khan, F. Al Ma'Mari, C. Kinane, S. Langridge, D. Ciudad, A. Bedoya-Pinto, L. Hueso, G. Teobaldi, V. K. Lazarov, D. Gilks, G. Burnell, B. J. Hickey, and O. Cespedes, Phys. Rev. B 90, 125311 (2014).

${ }^{26}$ K. Bairagi, A. Bellec, V. Repain, C. Chacon, Y. Girard, Y. Garreau, J. Lagoute, S. Rousset, R. Breitwieser, Y.-C. Hu, Y. C. Chao, W. W. Pai, D. Li, A. Smogunov, and C. Barreteau, Phys. Rev. Lett. 114, 247203 (2015).

${ }^{27}$ F. Al Ma'Mari, T. Moorsom, G. Teobaldi, W. Deacon, T. Prokscha, H. Luetkens, S. Lee, G. E. Sterbinsky, D. A. Arena, D. A. MacLaren, M. Flokstra, M. Ali, M. C. Wheeler, G. Burnell, B. J. Hickey, and O. Cespedes, Nature 524, 69 (2015).

${ }^{28}$ N. Atodiresei, V. Caciuc, P. Lazic, and S. Bluegel, Phys. Rev. B 84, 172402 (2011). 
${ }^{29}$ R. Friedrich, V. Caciuc, N. S. Kiselev, N. Atodiresei, and S. Bluegel, Phys. Rev. B 91, 115432 (2015).

${ }^{30}$ P. Lazic, V. Caciuc, N. Atodiresei, M. Callsen, and S. Bluegel, J. Phys.: Condens. Matter 26, 263001 (2014).

${ }^{31}$ O. Yabuhara, M. Ohtake, Y. Nukaga, and M. Futamoto, J. Phys.: Conf. Ser. 266, 012049 (2011).

${ }^{32}$ K. Wang, T. L. A. Tran, P. Brinks, J. G. M. Sanderink, T. Bolhuis, W. G. van der Wiel, and M. P. de Jong, Phys. Rev. B 88, 054407 (2013).
${ }^{33}$ J. Fraxedas, Y. J. Lee, I. Jimenez, R. Gago, R. M. Nieminen, P. Ordejon, and E. Canadell, Phys. Rev. B 68, 195115 (2003).

${ }^{34}$ B. H. Frazer, B. Gilbert, B. R. Sonderegger, and G. De Stasio, Surf. Sci. 537, 161 (2003).

${ }^{35}$ J. Vogel and M. Sacchi, J. Electron Spectrosc. Relat. Phenom. 67, 181 (1994).

${ }^{36}$ C. T. Chen, Y. Idzerda, H. J. Lin, N. V. Smith, G. Meigs, E. Chaban, G. H. Ho, E. Pellegrin, and F. Sette, Phys. Rev. Lett. 75, 152 (1995). 